\title{
Aspect angle dependence of the $E$ region irregularity velocity at large flow angles
}

\author{
R. A. Makarevich, ${ }^{1}$ A. V. Koustov, ${ }^{2}$ A. Senior, ${ }^{3}$ M. Uspensky, ${ }^{4}$ F. Honary, ${ }^{3}$ \\ and P. L. Dyson ${ }^{1}$ \\ Received 12 February 2007; revised 6 May 2007; accepted 13 July 2007; published 13 November 2007.
}

[1] We present the Doppler velocity observations of 1-m plasma waves in the auroral $E$ region by the Scandinavian Twin Auroral Radar Experiment (STARE) Norway VHF coherent radar in the context of simultaneous and coincident measurements of electron and ion drift velocities determined by the European Incoherent Scatter (EISCAT) tristatic radar facility. The measurements were performed in the afternoon sector (1500-

2000 MLT) at seven locations along the STARE radar beam 2 with different values of the magnetic off-perpendicular (aspect) angle $\alpha$ between $0.48^{\circ}$ and $2.63^{\circ}$ and at large angles with respect to the electron background drift $\left(\theta=55^{\circ}-90^{\circ}\right)$. It is demonstrated that the STARE line-of-sight velocity, normalized to the EISCAT-derived electron drift speed at large flow angles, exhibits a decrease with increasing aspect angle, and the rate of decrease is investigated as a function of the flow angle. We also compare the STARE velocity with the electron and ion drift velocity components along the STARE radar beam look direction and show that, at large aspect angles, the $E$ region velocity is correlated (anticorrelated) with the ion (electron) drift velocity component. The results are discussed in the contexts of the linear fluid theory of the modified two-stream plasma instability and the theory of anomalous collisions.

Citation: Makarevich, R. A., A. V. Koustov, A. Senior, M. Uspensky, F. Honary, and P. L. Dyson (2007), Aspect angle dependence of the $E$ region irregularity velocity at large flow angles, J. Geophys. Res., 112, A11303, doi:10.1029/2007JA012342.

\section{Introduction}

[2] The ionospheric plasma in the crossed electric $\mathbf{E}$ and geomagnetic $\mathbf{B}$ fields drifts with a velocity dependent on the electron and ion collision frequencies and hence on the altitude. In the $F$ region (above $\sim 120 \mathrm{~km}$ ), both plasma species drift with the $\mathbf{E} \times \mathbf{B}$ velocity $\mathbf{V}_{E}$, whereas in the $E$ region $(100-120 \mathrm{~km})$, where the ion-neutral collision frequency $\nu_{i}$ exceeds the ion gyrofrequency $\Omega_{i}$, the ion drift speed $V_{i 0}$ is several times smaller than that of the electrons $V_{e 0} \cong V_{E}$. Under strong electric field conditions, when $V_{e 0}$ exceeds the ion-acoustic speed $C_{s}$, the $E$-region plasma becomes unstable and sustains electrostatic waves or irregularities [Farley, 1963; Buneman, 1963]. The instability that gives rise to the fluctuations has been termed the modified two-stream or the Farley-Buneman instability (FBI). The linear fluid theory of the FBI predicts that in the ion frame of reference the FB waves are excited in a finite cone of flow angles $\theta<\theta_{0}$, given by $V_{e 0} \cos \theta_{0}=C_{s}$, and that at a

\footnotetext{
${ }^{1}$ Department of Physics, La Trobe University, Bundoora, Melbourne, Victoria, Australia.

${ }^{2}$ Institute of Space and Atmospheric Studies, University of Saskatchewan, Saskatoon, Saskatchewan, Canada.

${ }^{3}$ Department of Communication Systems, Lancaster University, Lancaster, UK.

${ }^{4}$ Space Research Unit, Finnish Meteorological Institute, Helsinki, Finland.

Copyright 2007 by the American Geophysical Union. 0148-0227/07/2007JA012342\$09.00
}

given angle $\theta$ the wave phase velocity $V_{p h}$ is proportional to the line-of-sight (1-o-s or los) component of the electron drift velocity $V_{e 0}^{C}=V_{e 0} \cos \theta: V_{p h}=\beta V_{e 0}^{C}$, where the coefficient $\beta$ is close to 1 [Fejer and Kelley, 1980]. Figure 1 illustrates the geometry of observations in the auroral $E$ region with the flow angle $\theta$ (the angle between wave propagation vector $\mathbf{k}$ and $\mathbf{V}_{e 0}$ ) shown by the black sector.

[3] The plasma density irregularities with a specific wavelength $\lambda_{i r r}$ can be detected by Doppler coherent radars with a frequency given by the Bragg condition, $f_{\text {rad }}=$ $c / 2 \lambda_{\text {irr }}$. The meter- and decameter-scale irregularities are routinely observed by the VHF and HF coherent radars both at small $\left(\theta<\theta_{0}\right)$ and large $\left(\theta>\theta_{0}\right)$ flow angles, inside and outside the FBI flow angle cone. The generation of waves at large flow angles is attributed to the energy transfer from the large-scale and/or inside-cone modes (see, e.g., the review by Farley [1985]). The phase velocity of outside-cone modes is significantly more difficult to predict and is often assumed to be equal to the velocity given by the linear theory, which was confirmed to some extent by numerical simulations [Keskinen et al., 1979] and radar observations [e.g., Nielsen and Schlegel, 1985; Nielsen et al., 2002].

[4] Small-scale irregularities are known to be highly magnetic field aligned. The FB waves are most easily excited perpendicular to the magnetic field direction and both their growth rate and phase velocity are fast-decreasing functions of the magnetic aspect angle $\alpha$ (the angle between the wave vector $\mathbf{k}$ and the plane perpendicular to the 


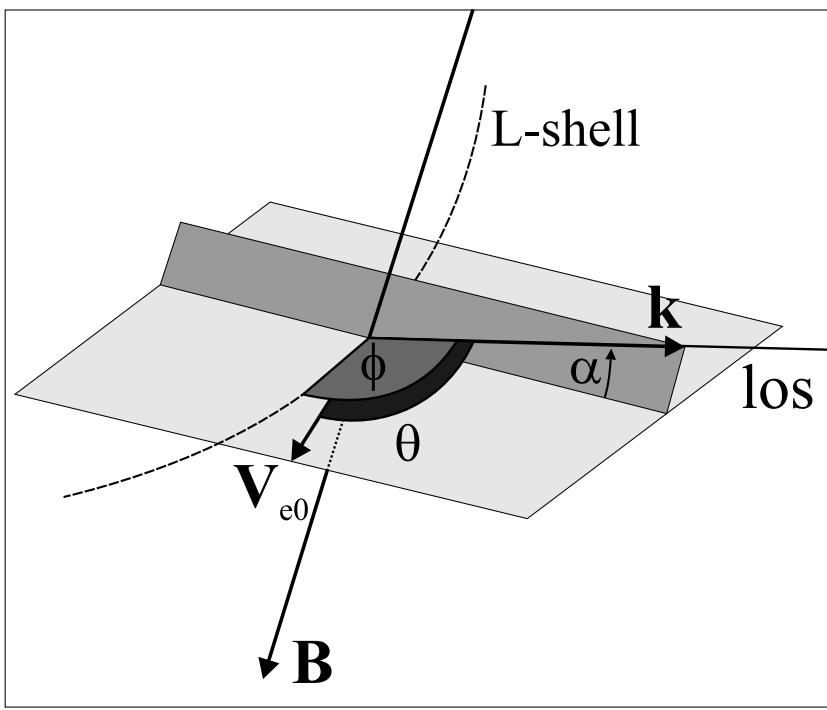

Figure 1. Geometry of observations in the auroral $E$ region. Shown are the magnetic field $\mathbf{B}$, the field-perpendicular plane, the electron background drift $\mathbf{V}_{e 0}$, the wave vector $\mathbf{k}$, the magnetic $L$ shell, the aspect angle $\alpha$, the flow angle $\theta$, and the $L$ shell angle $\phi$.

magnetic field $\mathbf{B}$ in Figure 1) so that no waves can be excited in the linear regime at $\alpha>1^{\circ}-1.5^{\circ}$ [e.g., Haldoupis, 1989]. Experimentally, it has been shown that the radar backscatter power decreases (attenuates) quickly with increasing distance between a given radar cell and the area with minimum magnetic aspect angle $\alpha$ [e.g., Nielsen, 1988]. The power attenuation rate varies with the aspect angle itself being larger at small $\alpha$, and appears to depend on the radar frequency as well, being larger at higher frequencies [Haldoupis, 1989].

[5] The $E$ region irregularity velocity has also been shown to decrease with the aspect angle $\alpha$, i.e., $V_{p h} \propto$ $\beta(\alpha)$ is a decreasing function of $\alpha$. At VHF, the velocity decrease was found to be consistent with the prediction of the linear fluid theory assuming that the electron-neutral collision frequency $\nu_{e}$ in the expression for phase velocity given by the linear fluid theory is replaced by an anomalous value, $\nu^{*}=6 \nu_{e}$ [Ogawa et al., 1980; Nielsen, 1986; Kustov et al., 1994]. It was argued that the above substitution is consistent with the notion that the irregularity phase velocity saturates at the ion-acoustic speed $C_{S}$ owing to the waveparticle interaction that effectively leads to an increase in the electron collision frequency [Sudan, 1983]. It is generally accepted that the above argument and the theory by Sudan [1983] provide a useful framework for interpreting the observations, but some theoretical reservations have been expressed as well [Haldoupis, 1989; Robinson and Honary, 1990; Foster et al., 1992]. Unlike backscatter power, the irregularity velocity attenuation rates were found to be similar at different frequency ranges [Ogawa et al., 1980; Nielsen, 1986; Foster et al., 1992; Makarevitch et al., 2002].

[6] The theory of Sudan [1983] contains one important prediction, i.e., that the anomalous collision frequency $\nu^{*}$ should be a decreasing function of the flow angle $\theta$ and that no increase should be observed at $\theta=\theta_{0}$. To date, most experimental studies of the aspect angle effects have concentrated on the observations at small flow angles, either close to the $\mathbf{V}_{e 0}$ direction or without considering explicitly different flow angles within a wide range of flow angles inside the cone. Moreover, it was often difficult to distinguish between the flow and aspect angle effects [e.g., Foster et al., 1992]. Makarevitch et al. [2002] have used the $L$ shell angle $\phi$ (the angle between the radar 1-o-s direction and magnetic $L$ shell direction, a predominant direction of the electron flow, see Figure 1) as a proxy for the flow angle. They have considered two ranges of $L$ shell angles and found that the decrease of the HF $E$ region velocity with the expected aspect angle was slower at larger $\phi$, an opposite result to the prediction of Sudan [1983].

[7] Makarevitch et al. [2002] also found that at the largest angles $\phi$ very little variation of the HF velocity with $\alpha$ was observed. Following earlier studies by Kohl et al. [1992] at VHF, by Moorcroft [1996] at UHF, and by Uspensky et al. [2001] at HF that suggested that the ion drift velocity contribution to the $E$ region irregularity velocity should be considered, Makarevitch et al. [2002] interpreted this observation as being consistent with the linear theory formula in the reference frame of neutrals at the largest flow angles, $\theta \cong 90^{\circ}$, where $V_{e 0}^{C} \cong 0$ and phase velocity $V_{p h} \cong V_{i 0}^{C}$ is independent of $\alpha$. More evidence on the importance of the ion motions at large flow angles has been presented at VHF [Uspensky et al., 2003, 2004; Makarevich et al., 2006a], at HF [Makarevitch et al., 2004] and at small flow angles and large aspect angles at HF [Milan et al., 2004].

[8] In the recent study by Makarevich et al. [2006b] the aspect angle dependence of the $E$ region irregularity velocity measured by the Scandinavian Twin Auroral Radar Experiment (STARE) Norway VHF radar has been studied using the coincident data on the electron drift velocity provided by the European Incoherent Scatter (EISCAT) UHF radar. Using the unique experimental opportunity provided by the special experiment design employed, it was possible to decouple the aspect angle effects from those of the flow angle. It was found that at intermediate flow angles $\theta=50^{\circ}-70^{\circ}$, that were most likely near the flow angle cone boundary $\theta \cong \theta_{0}$, the velocity decrease was consistent with the linear theory prediction without assuming anomalous collision frequencies. This result implies that the velocity decrease rate either is larger than previously thought [e.g., Ogawa et al., 1980; Nielsen, 1986] and/or is an increasing function of the flow angle in agreement with the theory of Sudan [1983]. Makarevich et al. [2006b] have also noted that the results of Makarevitch et al. [2002] showing the opposite effect for small velocities observed at large flow angles may have been affected by the ion motions (see also our section 3.2).

[9] The aim of this study is to investigate the aspect angle dependence of the $E$ region velocity at large flow angles, outside the FBI flow angle cone. We employ the data from the special experiments conducted in December 2004 using the STARE Norway coherent VHF radar and the EISCAT tristatic facility (a subset of these data was used by Makarevich et al. [2006b]. The specific objectives are (1) to determine whether the $E$ region irregularity velocity exhibits a decrease with the aspect angle at large flow angles $\theta>\theta_{0}$ and in particular close to $\theta=90^{\circ}$ using measurements at several locations with different aspect 


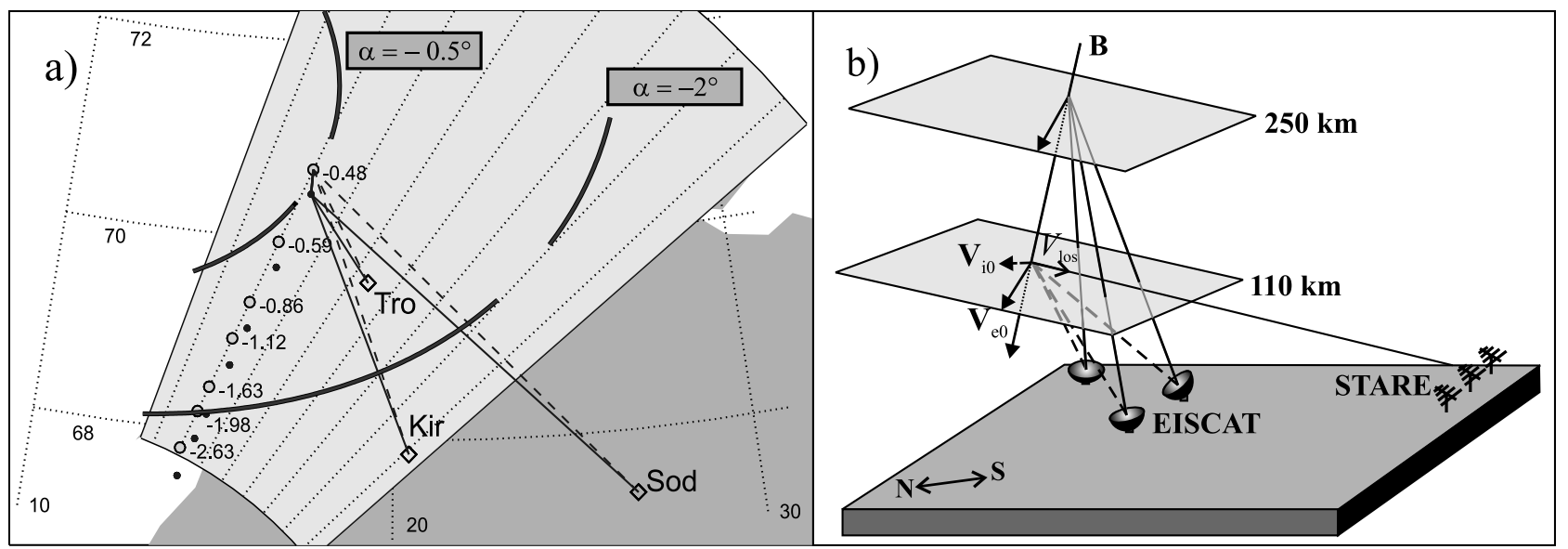

Figure 2. The experimental setup diagram showing (a) the geometry of observations in the horizontal plane and (b) the three-dimensional schematic view from the west. Figure 2a shows the field of view of the Scandinavian Twin Auroral Radar Experiment (STARE) Norway radar by the light grey sector with the nominal radar beam directions indicated by the dotted straight lines. The heavy curves represent the rectilinear aspect angle lines at $110 \mathrm{~km}, \alpha=-0.5^{\circ}$, and $\alpha=-2^{\circ}$. The diamonds are the locations of the European Incoherent Scatter (EISCAT) radar sites, and the solid (dashed) lines are the EISCAT radar beams intersecting at a particular location at $250 \mathrm{~km}(110 \mathrm{~km})$. The closed (open) circles show all locations of the EISCAT tristatic volume at $250 \mathrm{~km}(110 \mathrm{~km})$ that were located on the same field lines as the selected STARE radar cells along beam 2. The digits nearby indicate the aspect angle $\alpha$ for these cells. Figure $2 \mathrm{~b}$ is a three-dimensional view from the west. A magnetic field line $\mathbf{B}$ is shown by the inclined downward arrow. The field-perpendicular planes intersecting $\mathbf{B}$ at 250 and $110 \mathrm{~km}$ are shown by the rectangles. Also shown are the vector of the $E$ region ion (electron) drift velocity $\mathbf{V}_{i 0}\left(\mathbf{V}_{e 0}\right)$ measured by EISCAT at $110 \mathrm{~km}$ (inferred from the $F$ region EISCAT measurements of $\mathbf{V}_{i 0}$ at $250 \mathrm{~km}, \mathbf{V}_{i 0}^{250} \cong$ $\left.\mathbf{V}_{e 0}{ }^{250} \cong \mathbf{V}_{e 0}{ }^{110}\right)$, and the line-of-sight $E$ region velocity, $V_{\text {los }}$.

angles, (2) to estimate the velocity decrease rate for different flow angles and investigate whether it increases with the flow angle, and (3) to determine whether the ion drift velocity dominates over that of the electrons in the $E$ region phase velocity at large flow and aspect angles using the ion drift measurements on the same magnetic field line in the $E$ and $F$ regions.

\section{Observations}

\subsection{Experiment Setup}

[10] Figure 2 shows the experimental setup employed in this study. Figure 2a is a geographic map view and Figure $2 b$ is a three-dimensional schematic view from the west. The $E$ region Doppler velocity data employed in this study have been collected by the STARE Norway VHF radar at Midtsandan $\left(63.67^{\circ} \mathrm{N}, 10.73^{\circ} \mathrm{E} ; 140 \mathrm{MHz}\right)$. The $28.8^{\circ}$-wide radar's field-of-view (FoV) is shown by the light grey sector. The data were collected for 8 radar beams (from 1 to 8) separated by $3.6^{\circ}$ in azimuth, with the bore site direction at $26.2^{\circ} \mathrm{E}$. The nominal beam directions are shown by the dotted straight lines in Figure $2 \mathrm{a}$.

[11] The STARE radar used the information from the first two lags of the auto correlation function (ACF) to determine the Doppler 1-o-s velocity and backscatter power of the $E$ region echoes at $\sim 110 \mathrm{~km}$ [Greenwald et al., 1978; Nielsen, 1982; Uspensky et al., 2005]. By convention, the Doppler velocity is positive (negative) for echoes approaching (receding from) the radar site. The schematic view of Figure $2 \mathrm{~b}$ shows the magnetic field line $\mathbf{B}$, the fieldperpendicular planes at 250 and $110 \mathrm{~km}$, and a STARE beam intersecting the magnetic field line at $110 \mathrm{~km}$, with the positive 1-o-s velocity $V_{\text {los }}$ measured. The integration time was $20 \mathrm{~s}$. In terms of range, the STARE measurements were performed from 495 to $1245 \mathrm{~km}$ with $15-\mathrm{km}$ resolution. During the data postprocessing, echoes with low power $(\mathrm{SNR}<3 \mathrm{~dB})$ were excluded from further analysis.

[12] The electron drift velocity has been derived from the EISCAT radar measurements of the ion velocity in the $F$ region. The EISCAT UHF tristatic radar facility consists of three parabolic dish antennas with one site in Tromsø combining both transmitting and receiving capabilities and two remote site receivers at Kiruna and Sodankylä [Rishbeth and Williams, 1985]. The locations of the three radar sites are shown by the diamonds in Figure 2a. The EISCAT radar measures incoherent scatter ion-line spectrum, from which electron density, ion 1-o-s velocity, ion temperature, and electron/ion temperature ratio can be computed. Tristatic velocity is obtained from the three 1-o-s components using the method outlined in the work of Rishbeth and Williams [1985].

[13] Figure 2 shows schematically EISCAT tristatic measurements of the ion drift velocity in both the $E$ and $F$ regions. The three solid (dashed) lines originating from the radar sites are the EISCAT beams intersecting at the $F$ region height of $250 \mathrm{~km}$ ( $E$ region height of $110 \mathrm{~km}$ ). Both tristatic volumes are located on the same magnetic field line shown by the inclined arrow in Figure $2 \mathrm{~b}$ and short straight line in Figure $2 \mathrm{a}$ beginning (ending) on the closed (open) circle. The ion drift velocity vectors measured by EISCAT at 250 and $110 \mathrm{~km}$ are shown by the arrows in the respective 

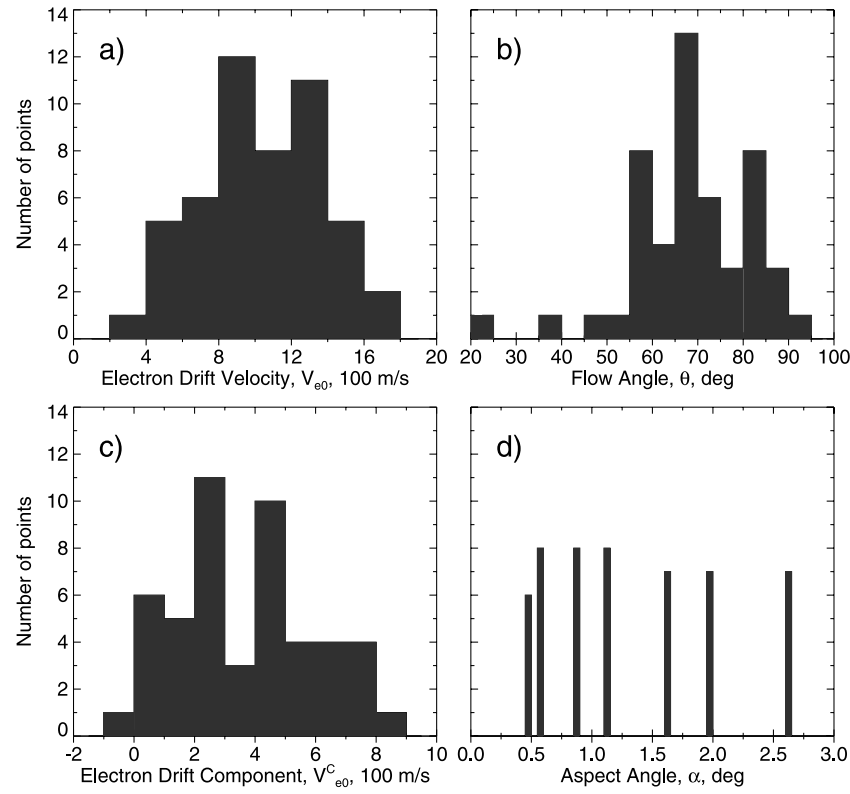

Figure 3. The distributions of (a) the electron drift speed, $V_{e 0}$, (b) the flow angle $\theta$, (c) the electron drift velocity component along the Norway radar beam $2, V_{e 0}^{C}=V_{e 0} \cos \theta$, and (d) the aspect angle $\alpha$ for all events considered.

field-perpendicular planes in Figure 2b. Since the background electric field (and hence the electron drift velocity $\left.\mathbf{V}_{e 0}\right)$ is approximately the same along $\mathbf{B}$, and since $\mathbf{V}_{i 0} \cong \mathbf{V}_{e 0}$ in the $F$ region, the ion drift velocity in the $F$ region at $250 \mathrm{~km}$ is a good proxy for the electron drift in the $E$ region at $110 \mathrm{~km}, \mathbf{V}_{i 0}^{F} \cong \mathbf{V}_{e 0}^{E}$. To compute l-o-s components, the tristatic velocity vectors are first projected onto the fieldperpendicular plane (these vectors are shown in Figure 2b) and then resolved along the beam direction.

[14] In this study, we consider the data collected on 9-12 December 2004 (at 1600-1800, 1500-1600, 1500-1600, and 1300-1600 UT, respectively; $\mathrm{MLT} \cong \mathrm{UT}+2)$ in a special campaign during which EISCAT operated in a scanning mode designed to derive the $E$ region electron drift velocity at 7 points located along the STARE Norway beam 2 (azimuth $17.2^{\circ} \mathrm{E}$ ). The EISCAT tristatic volume was located at $250 \mathrm{~km}$ (black dots in Figure 2a) and, when projected along the magnetic field lines down to the height of $110 \mathrm{~km}$, was successively coincident with the STARE range cells shown by the open circles in Figure $2 \mathrm{a}$ corresponding to rectilinear (geometrical) aspect angles shown by the digits near the open circles. These angles were calculated using the IGRF magnetic field model and assuming straight-line propagation from the STARE radar site to the center of the radar cell at $110 \mathrm{~km}$. Makarevich et al. [2006b] used slightly different nominal values for aspect angles as the southeastern corner instead of the center of each radar cell was considered. The interpolated locations with aspect angles of $\alpha=-0.5^{\circ}$ and $\alpha=-2^{\circ}$ in the STARE FoV are shown by the heavy curves in Figure $2 \mathrm{a}$.

[15] The duration of each scan was $30 \mathrm{~min}$ with the exception of 9 December 2004 when EISCAT also performed the tristatic ion drift measurements at $110 \mathrm{~km}$ with the total scan time of $60 \mathrm{~min}$. In this combined $E / F$ region mode both the electron and ion drift velocities were derived as shown in Figure $2 b$, with the ion drift velocity measured directly and the electron drift velocity inferred from projecting $\mathbf{V}_{i 0}^{F}$ down to $110 \mathrm{~km}$. Altogether, 12 scans have been performed, with the coincident STARE echoes observed during 8 scans. Both the EISCAT and STARE data were postintegrated over the EISCAT dwell time at each scan position ( $230 \mathrm{~s})$.

\subsection{Overview}

[16] Figure 3 presents a summary of the EISCAT measurements of the electron drift velocity, $\mathbf{V}_{e 0}$. It shows the histograms of the number of EISCAT observations with coincident STARE echoes versus (Figure 3a) the electron drift speed, $V_{e 0}$, (Figure $3 \mathrm{~b}$ ) the flow angle $\theta$, (Figure $3 \mathrm{c}$ ) the electron drift velocity component resolved along the STARE Norway beam 2 (azimuth $17.2^{\circ} \mathrm{E}$ ), $V_{e 0}^{C}=V_{e 0} \cos \theta$, and (Figure 3d) the aspect angle $\alpha$ in the centers of the STARE Norway radar cells located on the same magnetic field lines as the EISCAT tristatic volumes. A small number of observations ( 3 points) gave values outside the $x$ axis ranges. These had values $V_{e 0}=2060 \mathrm{~m} / \mathrm{s}, \theta=121^{\circ}$, and $V_{e 0}^{C}=$ $-699,1120 \mathrm{~m} / \mathrm{s}$, in Figures 3a, 3b, and 3c, respectively. The bin width in Figure $3 \mathrm{~d}$ was chosen to be small as geometrical aspect angles were exactly the same for the same scanning positions of EISCAT, Figure 2a. In this study, the vector from the radar cell to the radar site gives the positive direction, i.e., the vector components are positive (negative) when they are oriented toward (away from) the radar.

[17] The electron drift speed was above $400 \mathrm{~m} / \mathrm{s}$, in all but one case (in which case it was $350 \mathrm{~m} / \mathrm{s}$ ), in agreement with the well-known threshold value for the drift speed needed to excite the FB meter waves [e.g., Haldoupis et al. 1990]. More than half of the observations were under strongly driven conditions with $V_{e 0}$ in excess of $800 \mathrm{~m} / \mathrm{s}$. The typical flow angles, Figure $3 \mathrm{~b}$, were in the range $55^{\circ}-90^{\circ}$, i.e., at least some observations were outside the flow angle cone $\left(\theta>\theta_{0} \sim 65^{\circ}\right)$. Figure $3 \mathrm{c}$ shows a similar picture: the electron drift velocity component was between 0 and $800 \mathrm{~m} / \mathrm{s}$, meaning that some echoes observed were the primary, inside-cone waves for which $V_{e 0}^{C}>C_{s}$ and some were the secondary, outside-cone waves with $V_{e 0}^{C}<C_{s}$. A minimum in the number of echoes at $V_{e 0}^{C}=300-400 \mathrm{~m} / \mathrm{s}$, although statistically insignificant, is close to the nominal FB threshold drift speed. All aspect angles, Figure 3d, are well represented with at least 6 observations made for all aspect angle values and with 7 observation for all but one value.

\subsection{Irregularity Velocity Versus Flow Angle}

[18] We now turn our attention to the STARE observations of the $E$ region irregularity velocity. It is well accepted that it should vary with both the flow and aspect angles, and in Figures $4 \mathrm{a}$ and $4 \mathrm{~b}$ we show the STARE velocity as a function of the flow angle $\theta$ with the data points coded in the aspect angle $\alpha$ as indicated in the top left corner of Figure 4b. Only points at large aspect angles are shown in Figure $4 \mathrm{~b}$. The points with small aspect angles, $\alpha<1^{\circ}$ generally have larger velocities for $\theta<70^{\circ}$, as expected. Interestingly, this trend is not so obvious at large flow angles, $\theta>70^{\circ}$. The points with the largest aspect angles (squares) have small velocities $(<300 \mathrm{~m} / \mathrm{s})$, again in agree- 


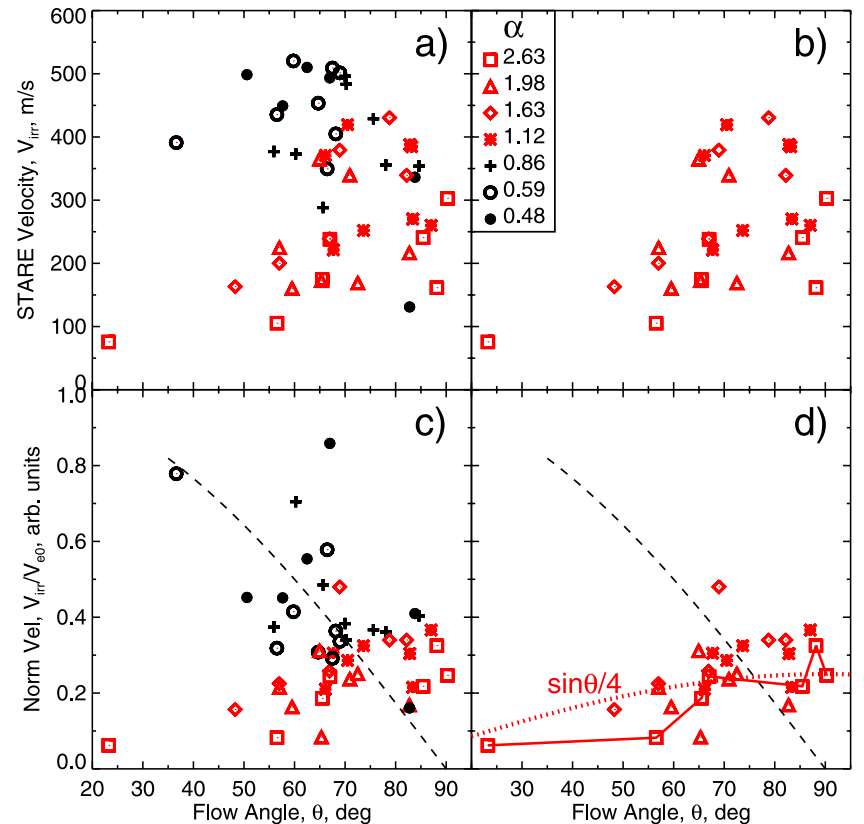

Figure 4. Flow angle variation of the $E$ region irregularity velocity (a) and (b) measured by STARE, $V_{i r r}$, (c) and (d) normalized to the electron drift speed, $V_{i r r} / V_{e 0}$. The data points are coded in aspect angle $\alpha$ according to the scheme in the top left corner of Figure $4 \mathrm{~b}$. The dashed line in Figures $4 \mathrm{c}$ and $4 \mathrm{~d}$ is the curve $V_{N}=\cos \theta$, and the dotted line in Figure $4 \mathrm{~d}$ is the curve $V_{N}=\sin \theta / 4$.

ment with the expected trend of the velocity decrease with aspect angle. On the other hand, no clear decrease of velocity with the flow angle is seen; velocities at large flow angles are only slightly smaller, and this may be due to aspect angle effects as the aspect angles are generally larger here (there are more points with large $\alpha$ at $\theta>70^{\circ}$ ).

[19] In addition to the flow and aspect angle effects, the irregularity velocity is expected to depend on the electron drift speed, $V_{e 0}$. As most of our data points are at large flow angles for which a simple proportionality is expected [e.g., Nielsen et al., 2002], one can normalize the irregularity velocity to the electron speed, $V_{N}=V_{i r r} / V_{e 0}$, and in Figures $4 \mathrm{c}$ and $4 \mathrm{~d}$ we show this normalized velocity. The expected cosine law curve, $V_{N}=\cos \theta$, is shown by the dashed line. The same (as in Figures $4 \mathrm{a}$ and $4 \mathrm{~b}$ ) general trend is observed here, with the points at small $\alpha$ located at larger velocities. These points are also close to the cosine curve. The points at large $\alpha$, on the other hand, are consistently below (above) the cosine trend at small (large) flow angles. In Figure $4 \mathrm{~d}$ we also show the trend $V_{N}=\sin \theta / 4$ (dotted line) suggested by earlier studies by Milan et al. [2004] and Makarevich et al. [2006a] (see also our section 3.1). One can notice from Figure $4 \mathrm{~d}$ that the data at the largest aspect angle $\alpha=2.63^{\circ}$ highlighted by the solid lines exhibit a general increase with the flow angle and appear to be consistent with this trend.

\subsection{Irregularity Velocity Versus Aspect Angle}

[20] The VHF velocity is known to depend on both the flow and aspect angles. The aspect angle dependence is believed to be quite strong, i.e., velocity decreases quickly with $\alpha$ [e.g., Nielsen, 1986]. One can expect therefore that velocity will be mostly dependent on the aspect angle $\alpha$ if the points from the same narrow range of $\theta$ are selected.

[21] Figure 5 shows the normalized velocity $V_{N}$ as a function of the aspect angle $\alpha$ for various flow angle ranges. The digits near the points indicate the electron drift component $V_{e 0}^{C}$ in $\mathrm{m} / \mathrm{s}$. To avoid overlapping of the data points with the same nominal aspect angle, the points were shifted horizontally by a small amount starting with the second point within the same group. The solid lines show the phase velocity variation with $\alpha$ as given by the linear theory of the FB instability with the collision frequencies taken from the MSISE-90 model run for the appropriate time and location $\left(\nu_{e}=32500 \mathrm{~s}^{-1}, \nu_{i}=765 \mathrm{~s}^{-1}\right)$. This theoretical trend is progressively smaller from Figures $5 \mathrm{a}$ to $5 \mathrm{~d}$ as it includes the cosine factor estimated for the center of the flow angle interval (e.g., for $\theta=75^{\circ}$ in Figure 5c).

[22] The data at the smallest flow angles in Figure 5a have the largest electron drift velocity components $V_{e 0}^{C}$ and agree well with the theoretical prediction. The data in Figure $5 \mathrm{~b}$ agree with the trend but only for the largest $V_{e 0}^{C}$. The disagreement is the largest for points with smallest $V_{e 0}^{C}$. In Figure $5 \mathrm{c}$ one can recognize a general decrease but the points are consistently above the theoretical curve. In Figure $5 \mathrm{~d}$ all points have $V_{e 0}^{C}<200 \mathrm{~m} / \mathrm{s}$ and lie above the solid line; no clear trend is observed here. One can conclude that the aspect angle dependence of the phase velocity appears to be different at various flow angles. It is consistent with the linear theory at small flow angles $\theta<60^{\circ}$, exhibits

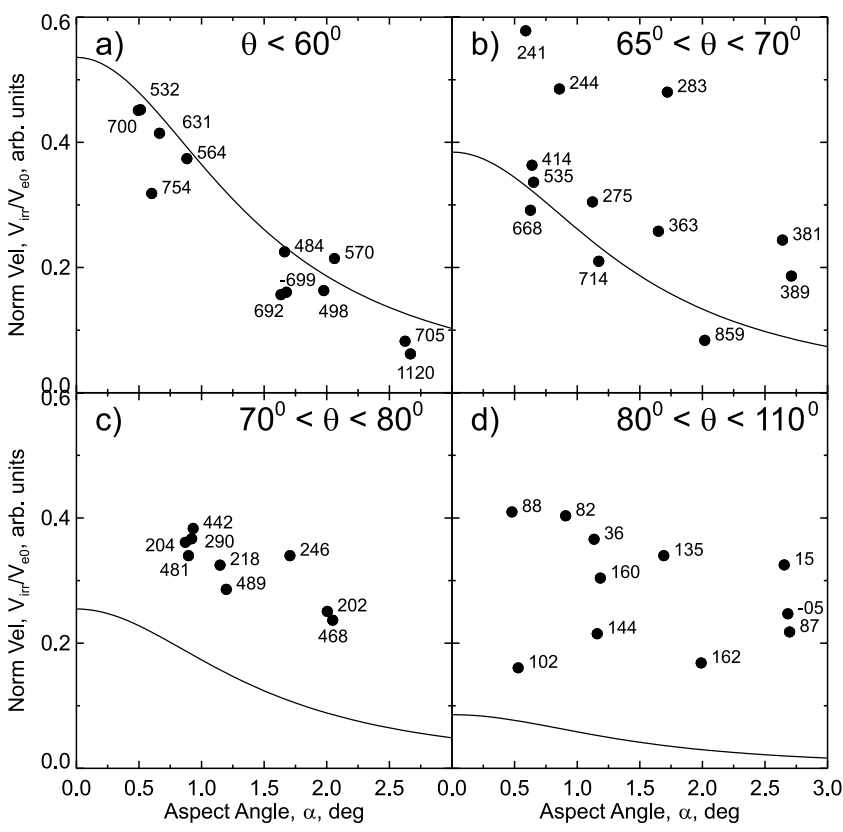

Figure 5. The normalized velocity $V_{N}$ versus the aspect angle $\alpha$ for different flow angles $\theta$ as indicated in the top right corner. The digits near the points indicate the electron drift velocity component along the STARE beam, $V_{e 0}^{C}$. The phase velocity variation with $\alpha$ given by the linear fluid theory with model collision frequency is shown by the solid line in each panel. 


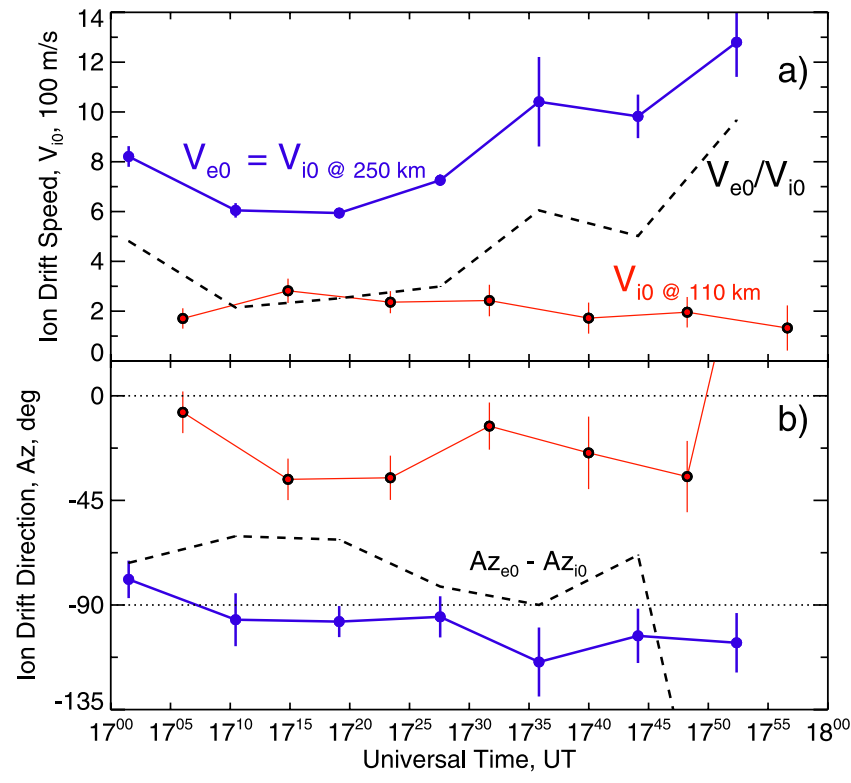

Figure 6. Time variation of the ion drift velocity (a) magnitude and (b) azimuth on 9 December 2004. The dashed lines in Figures $6 \mathrm{a}$ and $6 \mathrm{~b}$ are the ratio of the electron to the ion drift speed and the difference between azimuths, respectively.

some decrease with $\alpha$ while being larger than the linear theory prediction at $\theta=65^{\circ}-80^{\circ}$, and independent of $\alpha$ at the largest flow angles $\theta>80^{\circ}$. The aspect angle decrease rate of the VHF velocity also appears to be decreasing with the flow angle.

\subsection{Irregularity Velocity and Electron and Ion Drifts}

[23] In the previous two sections we normalized the irregularity velocity to the electron drift speed $V_{e 0}$ bearing in mind that at large flow angles the VHF velocity is believed to be proportional to $V_{e 0}$ [e.g., Moorcroft, 2002]. Analysis of the data from the combined $E / F$ region scan of EISCAT also provides an opportunity to investigate the relation between the $E$ region irregularity velocity and the ion drift velocity component, similar to the recent study by Makarevich et al. [2006a] that demonstrated that at the largest flow angles the $E$ region velocity may be representative of the ion drift component (rather than of that of the electrons).

[24] Figure 6 shows an example of the electron and ion drift velocity measurements by EISCAT. Figure 6a shows the magnitudes and Figure $6 \mathrm{~b}$ shows the directions (in geographic azimuth, East of geographic North). The electron-to-ion-speed ratio and the difference between azimuths are shown by the dashed lines in Figures $6 \mathrm{a}$ and $6 \mathrm{~b}$, respectively. The typical speed ratio was about 4 and the difference between directions was $70^{\circ}-90^{\circ}$, i.e., the ion drift vector was about 4 times smaller in length and rotated clockwise from the electron drift direction. By resolving the electron and ion drift vectors along the STARE Norway beam 2 direction one can derive the electron and ion drift components to compare with the 1-o-s $E$ region irregularity velocity measured by STARE (we have already used the resolved electron drift velocity component in Figure 5).
[25] Figure 7 shows the $E$ region irregularity velocity measured by STARE on 9 December 2004 versus the resolved (Figure 7a) electron and (Figure 7b) ion velocity components. Figures $7 \mathrm{c}$ and $7 \mathrm{~d}$ present similar comparisons for the entire data set. The ion drift components in Figure 7d were calculated from the EISCAT-derived electron drift velocity assuming that the ion drift velocity vector is 4 times smaller in length $\left(V_{i 0}=V_{e 0} / 4\right)$ and rotated by $90^{\circ}$ clockwise $\left(A z_{i 0}=A z_{e 0}-90^{\circ}\right)$ in rough agreement with Figure 6. The ion drift velocity component in Figure 7 was reversed as indicated by the minus sign in the $x$ axis label.

[26] For the data recorded on 9 December 2004, only the irregularity velocity at smaller aspect angles $\left(\alpha \leq 1.12^{\circ}\right)$ is close to the electron drift component in Figure 7a. At large aspect angles, the velocity is significantly depressed owing to the aspect angle effects with the depression increasing with the aspect angle, as expected. Interestingly enough, the irregularity velocity is close to the negative of the ion drift component at large aspect angles, Figure $7 \mathrm{~b}$. For the entire data set in Figure 7c, velocity is larger (smaller) than $V_{e 0}^{C}$ when $V_{e 0}^{C}$ is smaller (larger) than $\sim 400 \mathrm{~m} / \mathrm{s}$ at small aspect angles. The linear Pearson correlation coefficient [Wilks, 1995] for all points in Figure $7 \mathrm{c}$ is only 0.11 ; it is significantly larger for points with small aspect angles, 0.67. At large aspect angles, no clear trend is observed. The correlation coefficient for points with large aspect angles in Figure 7c is negative and increasing in magnitude with an increase in the lowest aspect angle considered; it is $-0.26,-0.34,-0.42$, and -0.80 for points with $\alpha \geq 1.12^{\circ}$, $1.63^{\circ}, 1.98^{\circ}$, and $2.63^{\circ}$. Remarkably, in Figure $7 \mathrm{~d}$, the irregularity velocity is close to the negative of the model ion drift velocity component, $-V_{i 0}^{C}$, at large aspect angles. The correlation coefficient is positive and increasing with an

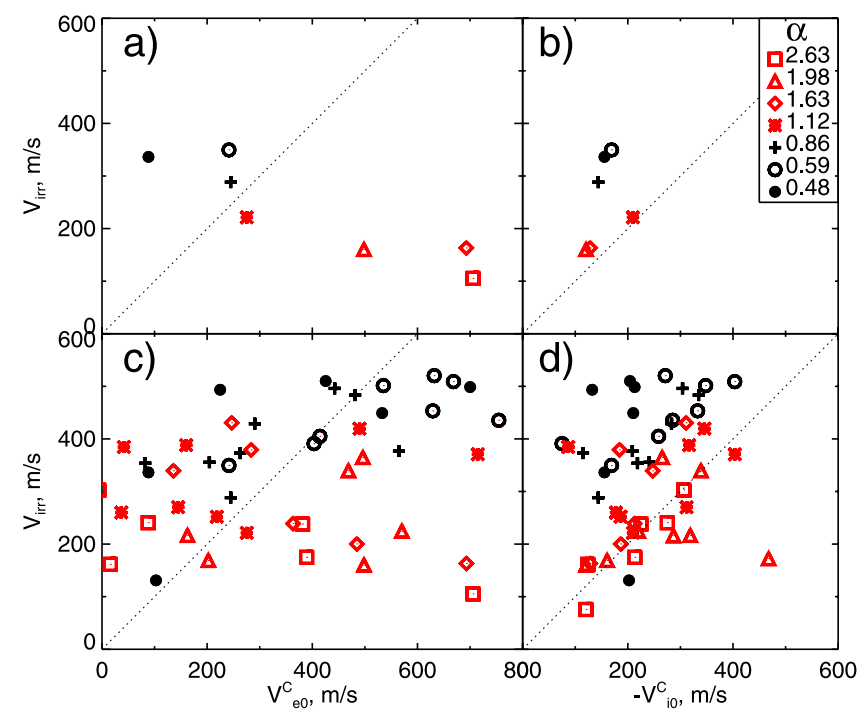

Figure 7. STARE velocity measured on 9 December 2004 versus (Figure 7a) the electron drift component $V_{e 0}^{C}$ and (Figure $7 \mathrm{~b}$ ) the negative of the ion drift component $V_{i 0}^{C}$. (c and d) Same as in Figures 7a and 7b but for all events and with the model ion drift component shown in Figure 7d. 
increase in the lowest aspect angle; it is $0.48,0.49,0.57$, and 0.80 for $\alpha \geq 1.12^{\circ}, 1.63^{\circ}, 1.98^{\circ}$, and $2.63^{\circ}$, respectively.

\section{Discussion}

[27] In this study we present observations of the $E$ region irregularity phase velocity measured by the STARE Norway VHF radar at several locations with different aspect angles from $\alpha=0.48^{\circ}$ to $2.63^{\circ}$ in conjunction with simultaneous and coincident data on the electron and, for one event, ion drift velocity component provided by the EISCAT tristatic facility. The relationship between the $E$ region irregularity and electron drift velocities has been extensively studied in the past including the STARE and EISCAT velocity comparisons similar to that employed in the present study [Nielsen and Schlegel, 1983, 1985; Kofman and Nielsen, 1990; Kustov and Haldoupis, 1992; Nielsen et al., 2002; Koustov et al., 2002; Uspensky et al., 2003, 2004, 2006]. All of the above studies, however, have used observations from the $\mathrm{CP}-1$ common mode of EISCAT. In this mode, position of tristatic volume is fixed and hence comparisons with the STARE velocity are only possible in one radar cell (located on the same magnetic field line as tristatic volume) with a fixed value of aspect angle of $\sim 1^{\circ}$.

[28] With respect to the aspect angle values, one has to bear in mind that in this study we used the nominal values computed as described in section 2.1 and that the real aspect angle values might have been different even at VHF frequencies owing to the altitude integration effects. The estimates show that typical variation due to changes in the electron density distribution at VHF is of the order of $0.1^{\circ}$ [Uspensky et al., 2003] and in the following we take this value for the uncertainty in the aspect angle.

[29] We demonstrated in Figure 3a that all of our data points refer to the strong electric field conditions with the FBI operational, $V_{e 0}>350 \mathrm{~m} / \mathrm{s}$. The flow angles computed, Figure $3 \mathrm{~b}$, indicated that the irregularities were observed both at small and large flow angles, inside and outside the FBI flow angle cone, respectively.

[30] According to the linear fluid theory of the FBI [e.g., Fejer and Kelley, 1980] developed for small flow angles, $\theta<\theta_{0}$, the irregularity phase velocity at a direction of wave propagation vector $\mathbf{k} \equiv \mathbf{k} / k$ is given by

$$
V_{p h}=\frac{\hat{\mathbf{k}} \cdot \mathbf{V}_{d}}{1+\Psi}+\hat{\mathbf{k}} \cdot \mathbf{V}_{i 0}=\frac{V_{d}^{C}}{1+\Psi}+V_{i 0}^{C},
$$

where $\mathbf{V}_{d}$ is the differential plasma drift velocity $\mathbf{V}_{d}=\mathbf{V}_{e 0}-$ $\mathbf{V}_{i 0}$ and the anisotropy factor $\Psi$ [Sahr and Fejer, 1996] is a function of aspect angle $\alpha$, collision frequencies of ions and electrons with neutrals $\left(\nu_{i}, \nu_{e}\right)$ and ion and electron gyro frequencies $\left(\Omega_{i}, \Omega_{e}\right)$ :

$$
\Psi=\frac{\nu_{e} \nu_{i}}{\Omega_{e} \Omega_{i}}\left(\cos ^{2} \alpha+\frac{\Omega_{e}^{2}}{\nu_{e}^{2}} \sin ^{2} \alpha\right)
$$

\subsection{Irregularity Velocity at Small and Intermediate Flow Angles}

[31] At small flow and aspect angles, both the ion drift velocity component $V_{i 0}^{C}$ and the anisotropy factor $\Psi$ are small and the phase velocity can be approximated as

$$
V_{p h} \cong \frac{V_{e 0}^{C}}{1+\Psi} \sim V_{e 0}^{C}
$$

[32] Experimental studies showed, however, that at small flow angles the irregularity velocity is significantly less than the electron drift component [Nielsen and Schlegel, 1983, 1985]. It has been proposed that the phase velocity is limited by the ion-acoustic speed, $V_{p h}=C_{s}$ [Nielsen and Schlegel, 1983] owing to the nonlinear processes [e.g., Sudan, 1983]. Since the ion-acoustic speed itself is an increasing function of the electron drift speed $V_{e 0}$, the phase velocity depends indirectly on the electron drift $V_{e 0}$ [Nielsen and Schlegel, 1985]. Later Nielsen et al. [2002] has derived an empirical formula for the phase velocity $V_{p h}=C_{s}(\cos \theta /$ $\left.\cos 40^{\circ}\right)^{\gamma}$, where the coefficient $\gamma$ is a decreasing function of the electron drift speed. Bahcivan et al. [2005] have recently suggested that the phase velocity is simply a 1-o-s component of the ion-acoustic speed, $V_{p h}=C_{s} \cos \theta$. All of these approaches imply that the velocity should be either a constant $\left(C_{S}\right)$ or a decreasing function of the flow angle.

[33] The phase velocity has also been shown to be a decreasing function of the aspect angle at small flow angles but the rate of decrease was slower than that given by equations (1) and (2). The velocity attenuation rate was found to be consistent with equation (1) with the substitution of $\nu_{e}$ in equation (2) by an enhanced value of $\nu^{*}=6 \nu_{e}$ and interpreted as being due to "anomalous" wave-particle collisions [Ogawa et al., 1980; Nielsen, 1986].

[34] In our observations at small aspect angles the phase velocity did exhibit a decrease with the flow angle which was roughly consistent with the cosine law curve, Figure 4c. At large aspect angles, a general VHF velocity increase was seen instead. The VHF velocity increase with the flow angle is unexpected and appears to be inconsistent with the previous VHF studies that reported a cosine-type decrease [e.g., Greenwald et al., 1978; Nielsen et al., 2002]. However, this result is consistent with the velocity increase with the flow angle at large aspect angles reported by Makarevitch et al. [2002] and Milan et al. [2004] at HF. Milan et al. [2004] proposed that at large aspect angles, the anisotropy factor $\Psi$ in equation (1) becomes very large and the phase velocity is to the first approximation proportional to the sine (ion drift velocity) component, with the coefficient dependent on the altitude and for their observations selected as 1/10. Later Makarevich et al. [2006a] showed that at $110 \mathrm{~km}$, the ion-to-electron-speed ratio is about $1 / 4$ and in Figure $4 d$ the flow angle variation at the largest aspect angle (solid lines) was consistent with this trend.

[35] At the smallest flow angles available, $\theta<60^{\circ}$, the irregularity velocity exhibited a decrease with the aspect angle which was consistent with the trend given by equation (1), Figure 5a, without any enhancement in the collision frequencies. This result is similar to that of the earlier analysis by Makarevich et al. [2006b] who considered the irregularity velocity normalized to the electron drift 


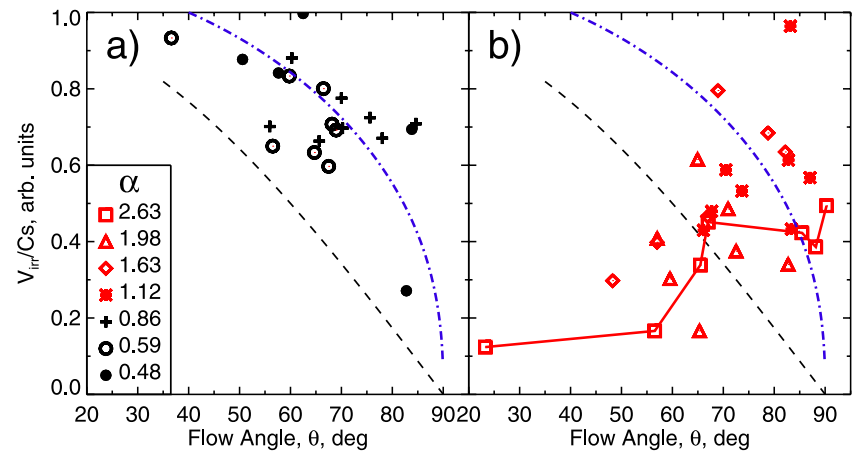

Figure 8. STARE velocity normalized to the ion-acoustic speed $C_{s}$ versus the flow angle $\theta$ for small (a) and large (b) aspect angles. The dashed line is the cosine law curve and the dash-dotted line is the trend given by the empirical formula of Nielsen et al. [2002].

velocity component $V_{e 0}^{C}$ and introduced a restriction $V_{e 0}^{C}>$ $500 \mathrm{~m} / \mathrm{s}$. One can easily see that both analyses produce essentially the same result, as the points with $V_{e 0}^{C}>500 \mathrm{~m} / \mathrm{s}$ lie close to the linear theory curve in Figures $5 \mathrm{a}$ and $5 \mathrm{~b}$. One should note, however, that this result is not inconsistent with the theory of anomalous collisions as most data points at $\theta<$ $60^{\circ}$ referred to the intermediate flow angles near the FBI cone boundary where no anomalous collisions are expected [Sudan, 1983].

\subsection{Irregularity Velocity at Large Flow Angles}

[36] Most of the data at large flow angles $\left(\theta>65^{\circ}\right)$ referred to the "overspeed" case [Uspensky et al., 2003], i.e., the irregularity velocity was larger than the electron drift component. This feature is inconsistent with the view that the irregularity velocity at large flow angles represents well the electron drift velocity, equation (3) [Kofman and Nielsen, 1990; Kustov and Haldoupis, 1992; Nielsen et al., 2002]. The other approach assumes that the irregularity velocity is related to the ion-acoustic speed $C_{s}$. Evidence has been presented that the irregularity velocity (1) is limited by the ion-acoustic speed at small flow angles $V_{p h}=C_{s}$ [Nielsen and Schlegel, 1983, 1985], (2) is described well by the empirical formula $V_{p h}=C_{s}\left(\cos \theta / \cos 40^{\circ}\right)^{\gamma}$ at small flow angles [Nielsen et al., 2002], and (3) is close to the 1-o-s component of the $C_{s}, V_{p h} \cong C_{s} \cos \theta$ at any flow angle [Bahcivan et al., 2005]. One can therefore investigate the flow angle dependence of the irregularity velocity normalized to $C_{s}$ instead of $V_{e 0}$ and determine whether this dependence is different at large aspect angles.

[37] Figure 8 shows the STARE velocity normalized to $C_{s}$ versus the flow angle in the same format as Figures $4 \mathrm{c}$ and $4 \mathrm{~d}$. The ion-acoustic speed for all events was estimated from the electron drift speed measurements by EISCAT at $250 \mathrm{~km}$ and an empirical formula similar to that of Nielsen and Schlegel [1985], viz. $C_{S}=A+B V_{e 0}^{2}$. The coefficients $A$ and $B$ were estimated by considering an extensive data set of EISCAT observations $\left(C_{s}\right.$ at $111 \mathrm{~km}$ versus $V_{e 0}=V_{i 0}$ at $278 \mathrm{~km}$ ) analogous to that in the work of Uspensky et al. [2006] and restricting it to the appropriate time sector (1200-1900 MLT). The best least squares fit coefficients were derived as $A=380 \mathrm{~m} \mathrm{~s}^{-1}, B=0.000155 \mathrm{~m}^{-1} \mathrm{~s}$.
[38] At small aspect angles, the normalized velocity is consistently above the dashed cosine curve implied by the model of Bahcivan et al. [2005], i.e., this model underestimates the irregularity velocity, the result consistent with that of Uspensky et al. [2006]. The empirical formula of Nielsen et al. [2002] with parameter $\gamma=0.4$ chosen for typical electron drift speeds of $1000 \mathrm{~m} / \mathrm{s}$ (Figure 3a), on the other hand, represents the data reasonably well. At large aspect angles, the points are above the cosine trend for large flow angles and, moreover, no clear decrease or perhaps even some increase is observed, e.g., at the largest aspect angle of $\alpha=2.63^{\circ}$. Both models disagree with observations at large aspect angles, Figure 8b. Recalling that at large $\alpha$ a significant disagreement was also observed between the irregularity velocity and the electron drift component, Figure $4 \mathrm{~d}$, one can conclude that none of the models that take into account the electron motions only explain reasonably well the flow angle dependence at large aspect angles.

[39] Milan et al. [2004] and Makarevitch et al. [2004] argued that as the flow angle increases, the electron drift component decreases as $\cos \theta$ whereas the ion drift component increases approximately as $\sin \theta$ so that one can expect the ion drifts to dominate at the largest flow angles and affect both the flow and aspect angle dependencies. Figure 4c showed that all points at $\theta>75^{\circ}$ are above the cosine law curve irrespectively of the aspect angle. Similarly, Figure 5 showed that the points at large $\theta$ and low $V_{e 0}^{C}$ were consistently above the linear theory trend. Moreover, at the largest flow angles, $\theta>80^{\circ}$, no decrease with the aspect angle was seen (Figure 5d). The latter observation is consistent with earlier observations by Makarevitch et al. [2002] at HF.

[40] From Figures $5 b-5 d$, it appears that the aspect angle attenuation rate at large flow angles is smaller than predicted by the linear theory and that it decreases with the flow angle. It may be incorrect, however, to interpret the observed experimental trends as being due to an increase in the effective collision frequency with the flow angle (an opposite effect to that implied by Sudan [1983]) as discussed below.

[41] According to equation (1), the phase velocity is a function of both the electron and ion drift components. The relative importance of the plasma species motions depends on the flow angle and the anisotropy factor. The factor $\Psi$ itself is a fast increasing function of the aspect angle and possibly an increasing function of the flow angle, indirectly through the anomalous collision frequency $\nu^{*}$, equation (2) [Sudan, 1983]. To determine whether $\nu^{*}$ and $\Psi$ depend indirectly on the flow angle, one needs to compare the function $\beta \equiv(1+\Psi)^{-1}$ for different flow angles. If the ion drift velocity is not zero in equation (1), a simple normalization to $V_{e 0}$ or $V_{e 0}^{C}$ would not yield the function $\beta$. From equation (1),

$$
\beta=\frac{1}{1+\Psi}=\frac{V_{p h}-V_{i 0}^{C}}{V_{d}^{C}},
$$

which reduces to $V_{p h} / V_{e 0}^{C}$ only in the limit $V_{i 0}^{C}=0$. Unfortunately, our attempts to estimate how the effective collision frequency/anisotropy factor varies with the flow angle by decoupling the direct effects of the flow angle and using equation (4) were largely inconclusive, as neither of the approaches (using measured $V_{e 0}^{C}$ and $\pm V_{i 0}^{C}$ for 
9 December 2004 and using measured $V_{e 0}^{C}$ and model $\pm V_{i 0}^{C}$ for the entire data set) showed any distinct features.

[42] The other limit of equation (1) is at the largest flow angles and/or at large aspect angles, where $\Psi \gg 1$. In this case, the phase velocity should be representative of the ion drift velocity component:

$$
V_{p h}=\frac{V_{d}^{C}}{1+\Psi}+V_{i 0}^{C} \cong V_{i 0}^{C}
$$

[43] This expectation was investigated by Makarevich et al. [2006a] who compared the STARE Finland velocity and the ion drift component $V_{i 0}^{C}$ measured by EISCAT in the $E$ region and found that the phase velocity was generally between the ion velocity components at 110 and $115 \mathrm{~km}$. In that study, however, the aspect angles were of the order of $1^{\circ}$ and the correlation between the irregularity and ion drift velocity was of the order of 0.6. In this study the aspect angles were up to $2.63^{\circ}$ and given a fast increase of $\Psi$ with $\alpha$, equation (2), one would expect the correlation to be more significant and less affected by the flow angle effects.

[44] Indeed, Figure 7 showed that the agreement between the irregularity and electron drift velocity was poor, in particular at large aspect angles $\alpha$, whereas the agreement between the irregularity and ion drift velocity at large $\alpha$ was quite reasonable both for the measured and model ion drift velocity components. The correlation in Figure $7 \mathrm{~d}$ was positive and increasing with the aspect angle reaching 0.8 at $\alpha=2.63^{\circ}$. The major difference between results of Makarevich et al. [2006a] and this study is that the ion drift component plotted in Figure 7 was reversed. In other words, the observed irregularity velocity at large $\alpha$ agreed well with the ion drift component in magnitude but was opposite in polarity.

[45] Interestingly, a similar result was reported by Kohl et al. [1992] who employed STARE and EISCAT data and used an equation analogous to equation (1) while assuming that the phase velocity is saturated at $C_{s}$ to infer the numerical values for $\Psi$ and $\mathbf{V}_{i 0}$. Kohl et al. [1992] concluded that equation (1) implies that the ions stream against the observed wave. Our analysis showed that for the phase velocity to be described well by equation (1) at large $\alpha$ the sign of the ion drift component should be reversed. One should note that all velocity polarities were consistent with those expected for this time of the day; the electron (ion) drifts were westward (poleward) and consistent with the westward plasma flow (poleward electric field) in the afternoon sector. The STARE Norway irregularity 1-o-s velocity was positive (toward the radar), which is consistent with the electron drift component sign and numerous previous studies [e.g., Kohl et al., 1992; Koustov et al., 2002; Uspensky et al., 2004]. Both Kohl et al. [1992] and Koustov et al. [2002] have also noted that their results might have been affected by the finite neutral winds at altitudes of $100-110 \mathrm{~km}$. However, the neutral wind magnitudes in the altitude range of interest are not expected to be considerably larger than $100 \mathrm{~m} / \mathrm{s}$ [e.g., Nozawa and Brekke, 1995], whereas the ion drift components in our observations were $100-400 \mathrm{~m} / \mathrm{s}$ and consistently close to the VHF velocity, Figure $7 \mathrm{~d}$. Clearly, this is an unexpected result, and the one that eludes explanation given our present understanding of the theory of electrojet irregularities.
[46] It should be emphasized though that the fact that at larger flow angles the $E$ region velocity (normalized to the electron drift speed/electric field) in Figure 5 exhibits a decrease with the aspect angle which is slower than that given by the linear theory and decreasing with the flow angle, does not necessarily mean that an increase with the flow angle occurs in the effective frequency $\nu^{*}$, as normalization to $V_{e 0}$ or $V_{e 0}^{C}$ may not fully account for the direct effects of the flow angle since the irregularity velocity can be affected by the ion motions as well.

\section{Summary}

[47] The $E$ region irregularity velocity measured by the STARE Norway VHF radar at flow angles $\theta=55^{\circ}-90^{\circ}$ was considered at 7 locations with aspect angles $\alpha$ between $0.48^{\circ}$ and $2.63^{\circ}$. The EISCAT tristatic system operated in a special scanning mode providing simultaneous and coincident data on the electron and (for one event) ion drift velocities in the $E$ region. The observations showed that

[48] 1. At large aspect angles $\alpha>1^{\circ}$, the $E$ region velocity variation with the flow angle was not consistent with the expected cosine variation and, moreover, it exhibited no decrease with the flow angle. At the largest aspect angle $\alpha=2.63^{\circ}$, the flow angle dependence was approximated by the sine of the flow angle multiplied by one quarter of the electron drift speed.

[49] 2. The aspect angle variation at the flow angles $\theta<$ $70^{\circ}$ and electron drift components exceeding $500 \mathrm{~m} / \mathrm{s}$ was found to be consistent with the prediction of the linear fluid theory without any enhancement in the electron collision frequency. At larger flow angles/smaller electron drift components, the velocity decrease rate was smaller than that given by the linear theory and decreasing with the flow angle so that at the largest flow angles $\theta>80^{\circ}$ no velocity decrease with the aspect angle was seen.

[50] 3. The $E$ region irregularity velocity at small aspect angles $\alpha<1^{\circ}$ was correlated reasonably well with the electron drift velocity component. At large aspect angles $\alpha>1^{\circ}$, an anticorrelation was observed instead, with the correlation coefficient magnitude increasing with the aspect angle. At $\alpha>1^{\circ}$, a substantial positive correlation was observed between the $E$ region velocity and the negative of the ion drift component with the correlation coefficient increasing with the aspect angle.

[51] 4. It was argued that the $E$ region velocity observations at large flow and/or aspect angles were likely to be affected by the ion motions and that without taking into account these effects it is impossible to conclude on the implications of the observed trends in the velocity aspect angle decrease rates (point 2) for the theory of anomalous collisions.

\section{Conclusion}

[52] The $E$ region irregularity phase velocity at large flow angles exhibits a decrease with the aspect angle. The rate of decrease is consistent with the prediction of the linear fluid theory of electrojet irregularities at the intermediate flow angles near the instability flow angle cone boundary. At larger flow angles, outside the flow angle cone, the velocity attenuation rate appears to be decreas- 
ing with the flow angle so that at the largest flow angles, velocity is largely independent of the aspect angle. At large aspect angles, the $E$ region phase velocity is close to the ion drift velocity component in terms of magnitude but opposite in polarity. The observed trends in the velocity decrease rate at large flow angles appear to be inconsistent with the anomalous collisions theory. However, the ion motion effects should be taken into account in order to study the anomalous collision frequency as a function of the flow angle in more detail.

[53] Acknowledgments. This research was partially supported by the Australian Research Council Discovery grant to R. A. M. (project DP0770366) and Natural Sciences and Engineering Research Council of Canada Discovery grant to A. V. K. The Scandinavian Twin Auroral Radar Experiment (STARE) system was operated jointly by the Max-PlanckInstitute for Aeronomy, Germany, and the Finnish Meteorological Institute, Finland, in cooperation with SINTEF, University of Trondheim, Norway. European Incoherent Scatter (EISCAT) is an international association supported by Finland (SA), France (Centre National de la Recherche Scientifique), Germany (Max-Planck-Institute Betreiben Grundlagenforschung), Japan (National Institute of Polar Research), Norway (Research Council of Norway), Sweden (Swedish Science Research Council), and the UK (Particle Physics and Astronomy Research Council). The authors are grateful to J. A. Davies of the Rutherford Appleton Laboratory for helpful comments on the experiment design.

[54] Amitava Bhattacharjee thanks Erhan Kudeki and another reviewer for their assistance in evaluating this paper.

\section{References}

Bahcivan, H., D. L. Hysell, M. F. Larsen, and R. F. Pfaff (2005), The $30 \mathrm{MHz}$ imaging radar observations of auroral irregularities during the JOULE campaign, J. Geophys. Res., 110, A05307, doi:10.1029/ 2004JA010975

Buneman, O. (1963), Excitation of field-aligned sound waves by electron streams, Phys. Rev. Lett., 10, 285-287.

Farley, D. T. (1963), A plasma instability resulting in field-aligned irregularities in the ionosphere, J. Geophys. Res., 68, 6083-6093.

Farley, D. T. (1985), Theory of equatorial electrojet plasma waves: New developments and current status, J. Atmos. Terr. Phys., 47, $729-744$.

Fejer, B. G., and M. C. Kelley (1980), Ionospheric irregularities, Geophys. Rev., 18, 401-454.

Foster, J. C., D. Tetenbaum, C. F. del Pozo, J.-P. St-Maurice, and D. R. Moorcroft (1992), Aspect angle variations in intensity, phase velocity and altitude for high-latitude $34 \mathrm{~cm} E$ region irregularities, J. Geophys. Res., 97, 8601-8617.

Greenwald, R. A., W. Weiss, E. Nielsen, and N. R. Thomson (1978), STARE: A new radar auroral backscatter in northern Scandinavia, Radio Sci., 13, 1021-1029.

Haldoupis, C. (1989), A review on radio studies of auroral $E$ region ionospheric irregularities, Ann. Geophys., 7, 239-258.

Haldoupis, C., E. Nielsen, and K. Schlegel (1990), Dependence of radar auroral scattering cross section on the ambient electron density and the destabilizing electric field, Ann. Geophys., 8, 195-212.

Keskinen, M. J., R. N. Sudan, and R. L. Ferch (1979), Temporal and spatial power spectrum studies of numerical simulations of type 2 gradient drift irregularities in the equatorial electrojet, J. Geophys. Res., 84, 14191430.

Kofman, W., and E. Nielsen (1990), STARE and EISCAT measurements: Evidence for the limitation of STARE Doppler velocity observations by the ion acoustic velocity, J. Geophys. Res., 95, 19,131-19,136.

Kohl, H., E. Nielsen, K. Rinnert, and K. Schlegel (1992), EISCAT results during the ROSE campaign and comparison with STARE measurements, J. Atmos. Terr. Phys., 54, 733-739.

Koustov, A. V., et al. (2002), Velocities of auroral coherent echoes at 12 and $144 \mathrm{MHz}$, Ann. Geophys., 20, 1647-1662.

Kustov, A. V., and C. Haldoupis (1992), Irregularity drift velocity estimates in radar auroral backscatter, J. Atmos. Terr. Phys., 54, 415-423.

Kustov, A. V., M. V. Uspensky, G. J. Sofko, J. A. Koehler, and J. Mu (1994), Aspect angle dependence of the radar aurora Doppler velocity, J. Geophys. Res., 99, 2131-2144.

Makarevitch, R. A., A. V. Koustov, G. J. Sofko, D. André, and T. Ogawa (2002), Multifrequency measurements of HF Doppler velocity in the auroral E region, J. Geophys. Res., 107(A8), 1212, doi:10.1029/ 2001JA000268.
Makarevitch, R. A., F. Honary, and A. V. Koustov (2004), Simultaneous HF measurements of $E$ - and $F$-region Doppler velocity at large flow angles, Ann. Geophys., 22, 1177-1188.

Makarevich, R. A., F. Honary, V. S. C. Howells, A. V. Koustov, S. E. Milan, J. A. Davies, A. Senior, I. W. McCrea, and P. L. Dyson (2006a), A first comparison of irregularity and ion drift velocity measurements in the $E$ region, Ann. Geophys., 24, 2375-2389.

Makarevich, R. A., A. Senior, A. V. Koustov, M. V. Uspensky, F. Honary, and P. L. Dyson (2006b), A study of aspect angle effects in the $E$-region irregularity velocity using multi-point electric field measurements, Geophys. Res. Lett., 33, L21102, doi:10.1029/2006GL027740.

Milan, S. E., M. Lester, T. K. Yeoman, T. R. Robinson, M. V. Uspensky, and J.-P. Villain (2004), HF radar observations of high-aspect angle backscatter from the E-region, Ann. Geophys., 22, 829-847.

Moorcroft, D. R. (1996), Flow angle effects in $E$ region 398-MHz auroral backscatter at small aspect angle, J. Geophys. Res., 101(A6), 13,379-13,386.

Moorcroft, D. R. (2002), Outstanding issues in the theory of radar aurora: Evidence from the frequency dependence of spectral characteristics, J. Geophys. Res., 107(A10), 1301, doi:10.1029/2001JA009218.

Nielsen, E. (1982), The STARE system and some of its applications, in The IMS Source Book: Guide to the International Magnetospheric Study Date Analysis, edited by D. J. Southwood and C. T. Russell, pp. 213-224, AGU, Washington, D.C.

Nielsen, E. (1986), Aspect angle dependence of mean Doppler velocities of 1-m auroral plasma waves, J. Geophys. Res., 91, 10,173-10,177.

Nielsen, E. (1988), Aspect angle dependence of backscatter intensity of 1-m auroral plasma waves, J. Geophys. Res., 93, 4119-4124.

Nielsen, E., and K. Schlegel (1983), A first comparison of STARE and EISCAT electron drift velocity measurements, J. Geophys. Res., 88, 5745-5750.

Nielsen, E., and K. Schlegel (1985), Coherent radar Doppler measurements and their relationship to the ionospheric electron drift velocity, J. Geophys. Res., 90, 3498-3504.

Nielsen, E., C. F. del Pozo, and P. J. S. Williams (2002), VHF coherent radar signals from the $E$ region ionosphere and the relationship to electron drift velocity and ion acoustic velocity, J. Geophys. Res., 107(A1), 1012, doi:10.1029/2001JA900111.

Nozawa, S., and A. Brekke (1995), Studies of the $E$ region neutral wind in the disturbed auroral ionosphere, J. Geophys. Res., 100, 14,717-14,734.

Ogawa, T., B. B. Balsley, W. L. Ecklund, D. A. Carter, and P. E. Johnston (1980), Aspect angle dependence of irregularity phase velocities in the auroral electrojet, Geophys. Res. Lett., 7, 1081-1084.

Rishbeth, H., and P. J. S. Williams (1985), The EISCAT ionospheric radar: The system and its early results, Q. J. R. Astron. Soc., 26, 478-512.

Robinson, T. R., and F. Honary (1990), A resonance broadening kinetic theory of the modified two-stream instability: Implications for radar auroral backscatter experiments, J. Geophys. Res., 95, 1073-1085.

Sahr, J., and B. G. Fejer (1996), Auroral electrojet plasma irregularity theory and experiment: A critical review of present understanding and future directions, J. Geophys. Res., 101, 26,893-26,909.

Sudan, R. N. (1983), Unified theory of type 1 and type 2 irregularities in the equatorial electrojet, J. Geophys. Res., 88, 4853-4860.

Uspensky, M. V., A. V. Koustov, P. Eglitis, A. Huuskonen, S. E. Milan, T. Pulkkinen, and R. Pirjola (2001), CUTLASS HF radar observations of high-velocity E-region echoes, Ann. Geophys., 19, 411-424.

Uspensky, M., A. Koustov, P. Janhunen, R. Pellinen, D. Danskin, and S. Nozawa (2003), STARE velocities: Importance of off-orthogonality and ion motions, Ann. Geophys., 21, 729-743.

Uspensky, M., A. Koustov, P. Janhunen, E. Nielsen, K. Kauristie, O. Amm, R. Pellinen, H. Opgenoorth, and R. Pirjola (2004), STARE velocities: 2. Evening westward electron flow, Ann. Geophys., 22, $1077-1091$

Uspensky, M., et al. (2005), Multipulse and double-pulse velocities of Scandinavian Twin Auroral Radar Experiment (STARE) echoes, Radio Sci., 40, RS3008, doi:10.1029/2004RS003151.

Uspensky, M. V., A. V. Koustov, and S. Nozawa (2006), STARE velocity at large flow angles: Is it related to the ion acoustic speed?, Ann. Geophys., $24,873-885$.

Wilks, D. S. (1995), Statistical Methods in the Atmospheric Sciences, Elsevier, New York.

P. L. Dyson and R. A. Makarevich, Department of Physics, La Trobe University, Bundoora, Melbourne, Vic 3086, Australia. (r.makarevich@ latrobe.edu.au)

F. Honary and A. Senior, Department of Communication Systems, Lancaster University, InfoLab21, Lancaster LA1 4WA, UK.

A. V. Koustov, Institute of Space and Atmospheric Studies, University of Saskatchewan, 116 Science Place, Saskatoon, SK, Canada S7N 5E2.

M. Uspensky, Space Research Unit, Finnish Meteorological Institute, P.O. Box 503, Helsinki FIN-00101, Finland. 